Cite this: Org. Biomol. Chem., 2016, 14,3404

\section{Enzymatic reduction of acetophenone derivatives with a benzil reductase from Pichia glucozyma (KRED1-Pglu): electronic and steric effects on activity and enantioselectivity $\dagger$}

\author{
Martina L. Contente, ${ }^{a}$ Immacolata Serra, ${ }^{\text {a }}$ Luca Palazzolo, ${ }^{\text {b }}$ Chiara Parravicini, ${ }^{\text {b }}$ \\ Elisabetta Gianazza, ${ }^{\mathrm{b}}$ Ivano Eberini, ${ }^{\mathrm{b}}$ Andrea Pinto, ${ }^{\mathrm{c}}$ Benedetta Guidi, ${ }^{\mathrm{d}}$ \\ Francesco Molinari ${ }^{a}$ and Diego Romano*a
}

\begin{abstract}
A recombinant ketoreductase from Pichia glucozyma (KRED1-Pglu) was used for the enantioselective reduction of various mono-substituted acetophenones. Reaction rates of meta- and para-derivatives were consistent with the electronic effects described by $\sigma$-Hammett coefficients; on the other hand, enantioselectivity was determined by an opposite orientation of the substrate in the binding pocket. Reduction of ortho-derivatives occurred only with substrates bearing substituents with low steric impact (i.e., $\mathrm{F}$ and $\mathrm{CN}$ ). Reactivity was controlled by stereoelectronic features ( $\mathrm{C}=\mathrm{O}$ length and charge, shape of LUMO frontier molecular orbitals), which can be theoretically calculated.
\end{abstract}

Received 8th January 2016, Accepted 29th February 2016

DOI: $10.1039 / \mathrm{c} 60 \mathrm{~b} 00047 \mathrm{a}$

www.rsc.org/obc cular dynamics simulations suggest that low reactivity of orthoderivatives is mostly due to unfavourable intramolecular interactions between the ortho carbons of the aromatic group and the nucleophilic nitrogen, as well as between the stereocenter and the enzymatic oxyanion. Moreover, the analysis of the solvent accessible surfaces of the hydrogens of the phenyl ring explains why para and meta substituents are good substrates, whereas ortho-substituted substrates were not accepted. ${ }^{5}$

Acetophenone derivatives are generally reduced with opposite enantioselectivity depending on the enzyme employed. An ADH from Rhodococcus erythropolis proved efficient in the reduction of a number of mono-, di- and tri-substituted acetophenones, giving always the corresponding (S)-1-phenylethanols (Prelog rule); ${ }^{6}$ the same stereopreference was observed with other ADHs, i.e., from Ralstonia sp. ${ }^{7}$ and from Thermoanaerobacter sp. ${ }^{8}$ A general preference for the Prelog rule was also detected in a screening with 24 recombinant KRs tested on thirteen mono-substituted acetophenones. ${ }^{9}$ Conversely, optically pure $(R)-1$-phenylethanol can be prepared by the reduction of unsubstituted acetophenone using specific enzymes, such as LK-ADH from Lactobacillus kefir, ${ }^{10}$ PF-ADH from Pseudomonas fluorescens ${ }^{11}$ and carbonyl reductase from Candida magnoliae (CMCR). ${ }^{12}$

Systematic studies on the effect of ring-substituents on enzymatic catalytic efficiency are quite rare. In fact, steric hindrance and electronic properties of acetophenone derivatives are largely dependent on the type and position of the substituent, thus affecting their overall reactivity, no matter if a chemo- or a bio-catalyst is involved. A number of mono-substi- 
tuted acetophenone derivatives were studied using Candida tenuis xylose reductase (CtXR), which showed, in all the cases, preference for the formation of (S)-1-phenylethanols. ${ }^{13}$ For this enzyme, $\sigma$-Hammett coefficients of para- and meta-substituents were in good correlation with the reaction velocities, indicating again that enzyme-substrate binding has a subordinate influence on the $C t$ XR catalytic efficiency on these substrates. Interestingly, ortho-derivatives were sometimes reduced with higher rates, indicating the occurrence of a beneficial mode of binding with favourable electronic interactions between the substrate and the enzyme. An interesting effect of the nature of the para-substituents was observed with ADH from Lactobacillus kefir and Thermoanaerobacter sp., by which acetophenones with neutral para-substituents were efficiently and selectively reduced, whereas those with ionizable substituents could not be reduced. ${ }^{14} \mathrm{~A}$ recombinant short-chain dehydrogenase from Saccharomyces cerevisiae showed excellent (S)enantioselectivity with a series of mono-substituted acetophenones, irrespective of the nature of the substituent. Notably, in the reduction of ethyl 2-oxo-2-phenylacetate, the enantioselectivity of this enzyme was strongly affected by the presence of substituents on the aromatic ring: with any substituent on the phenyl ring, the $(R)$-alcohol was produced, whereas the unsubstituted ethyl 2-oxo-2-phenylacetate gave the (S)alcohol. ${ }^{15}$ These results show that not only reactivity, but also enantioselectivity may be influenced by the presence of substituents on the aromatic ring.

In this study we have systematically investigated the effect of the steric and electronic properties of the substituent on the reduction of different mono-substituted acetophenones catalysed by a new recombinant ketoreductase (KRED1-Pglu) ${ }^{16,17}$

\section{Results and discussion}

The recombinant benzil ketoreductase KRED1-Pglu was isolated from the non-conventional yeast Pichia glucozyma; ${ }^{16}$ whole cells of $P$. glucozyma enantioselectively reduced different aromatic ketones, ${ }^{18-20}$ including acetophenone, with a marked preference for the formation of (S)-1-phenylethanol; ${ }^{21,22}$ interestingly, KRED1-Pglu was isolated from the same yeast reduced acetophenone as a recombinant protein with opposite enantioselectivity. KRED1-Pglu was initially investigated using different para-substituted acetophenone derivatives (1a-1c and $\mathbf{1 e - 1 h}$ ); acetophenone (1d) was considered as a reference (Table 1). The substrates were transformed with very different rates, except for 4'-aminoacetophenone 1a (entry 1, Table 1), which was the only substrate not to be processed. Reduction followed the Prelog rule for all the substituted acetophenones, giving the corresponding (S)-1-phenylethanols; most of the biotransformations occurred with high enantioselectivity, with the exception of 4'-methoxyacetophenone $\mathbf{1 b}$ (entry 2, Table 1), which was reduced with only $60 \%$ ee. The observed stereopreference is opposed to the one obtained with acetophenone 1d, which was reduced to $(R)$-1-phenylethanol 2 d with $95 \%$ ee. KRED1-Pglu was also used for the reduction of a set of meta-
Table 1 Reduction of para-substituted acetophenones with KRED1Pglu

\begin{tabular}{llllll}
\hline & & & \\
Entfer pH $8.0 / 30^{\circ} \mathrm{C}$
\end{tabular}

substituted acetophenones (Table 2), having the same substituents of the para-acetophenones previously tested (1i-1o). In this case, all the substrates did react, including the amino derivative 1i. All the meta-derivatives were reduced to the corresponding (S)-1-phenylethanols with high-to-excellent enantioselectivity. Notably, $3^{\prime}$-methoxyacetophenone $\mathbf{1 j}$ was reduced to enantiopure $(S)-2 \mathbf{j}$, whereas the corresponding paraderivative $\mathbf{1 b}$ showed moderate enantioselectivity.

The $\sigma$-Hammett coefficients were used to assess the influence of the nature of the para- and meta-substituents on the kinetics of the hydride transfer to acetophenone. The initial rates were plotted against the $\sigma$-values of the substituents; $\sigma_{\text {para }}$ and $\sigma_{\text {meta }}$ were independently correlated (Fig. 1). $\sigma_{\text {para }}{ }^{-}$ Hammett coefficients were in excellent correlation with the reaction rates, showing that the activity of KRED1-Pglu was predominantly dependent on the electronic properties, while the substrate size and/or hydrophobicity had negligible effects. The reaction rate was accelerated by the electron withdrawing groups $(\rho>0)$, as a consequence of the negative charge in the transition state caused by the attack of the hydride on the carbonyl group.

Table 2 Reduction of meta-substituted acetophenones with KRED1Pglu

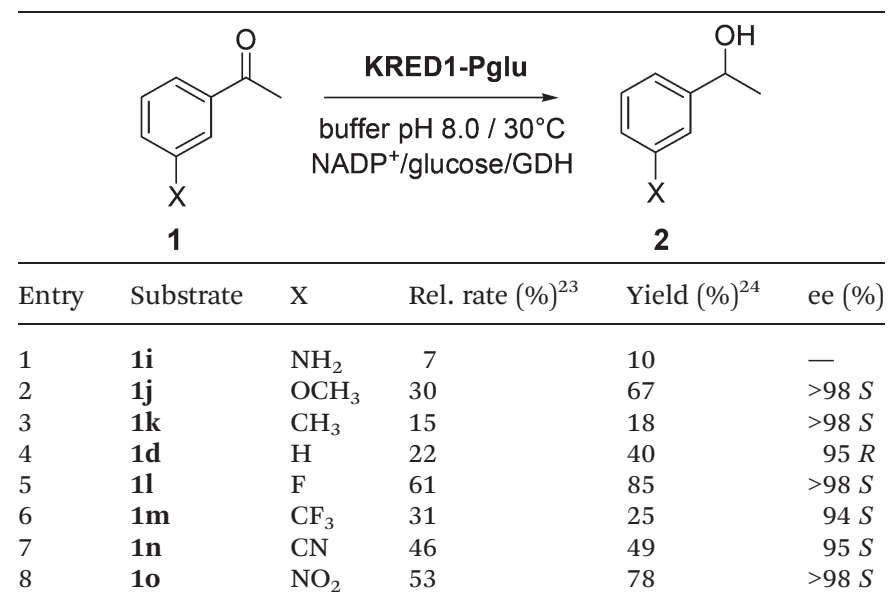



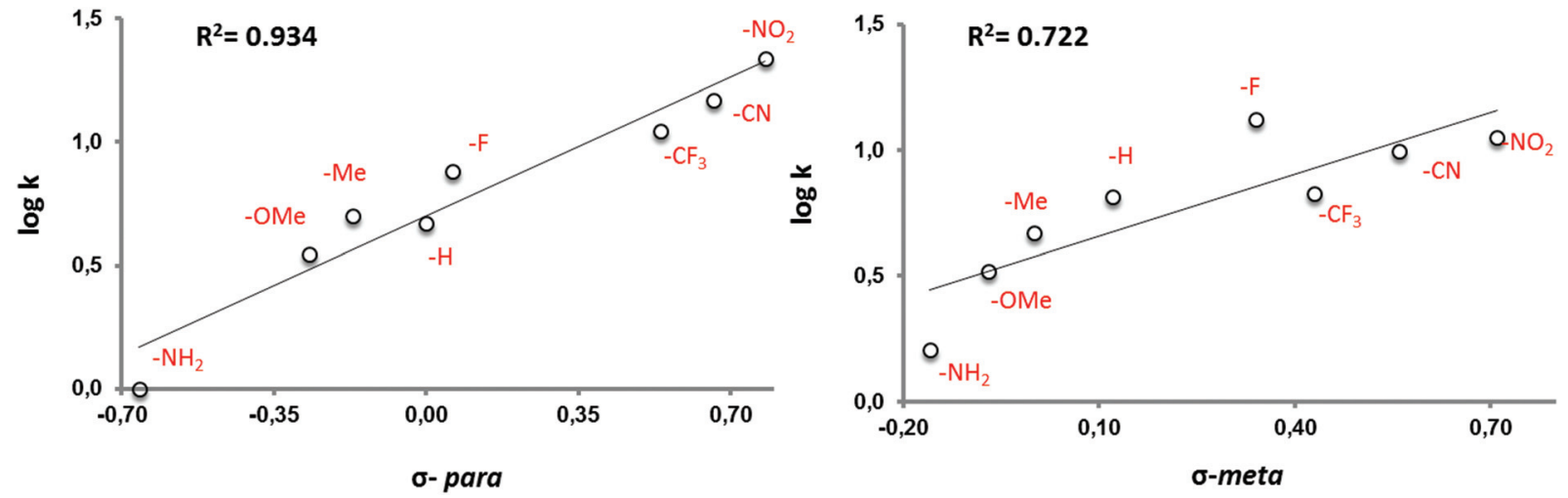

Fig. 1 Correlation between activity and the Hammett $\sigma$-values.

In order to evaluate the electronic features of the tested substrates, a geometry optimization in the gas phase with an approach based on density functional theory (DFT/B3LYP/6$31 \mathrm{G}^{*}$ ) was performed, resulting in prediction of the $\mathrm{C}=\mathrm{O}$ lengths and of the Mulliken derived charges for the $\mathrm{C}$ atom involved in the hydride attack of the substrates. A further characterization was carried out through the analysis of the lowest unoccupied molecular orbitals (LUMO) in the investigated substrates. The activity of para- and meta-derivatives was also correlated with the electronic charge density distribution of the $\mathrm{C}=\mathrm{O}$ bond (Fig. 2) and $\mathrm{C}=\mathrm{O}$ length (Fig. 3). These factors have been previously used as parameters for correlating the activity of ADHs on different mono-substituted acetophenones. ${ }^{12,13}$ A high correlation between activity and electronic features of the substituents was found with para-derivatives, whereas a lower correlation could be detected with metaderivatives, as also seen with other enzymes. ${ }^{12}$ This behaviour is different from what was observed with ADHs from
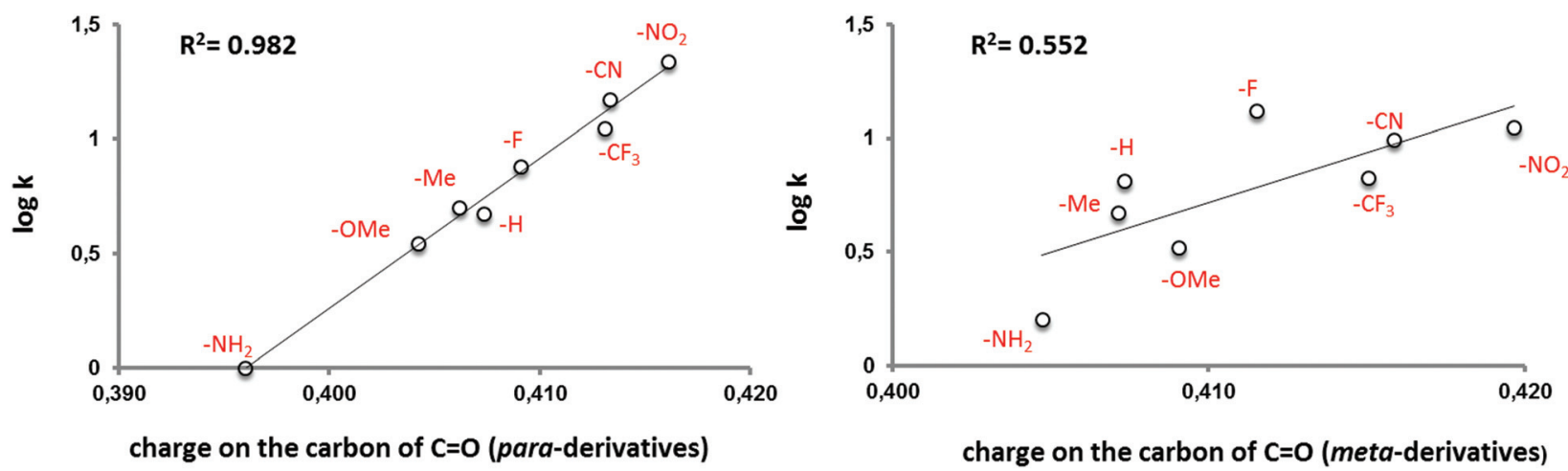

Fig. 2 Correlation between activity and charge on the carbon of the $\mathrm{C}=\mathrm{O}$ bond.
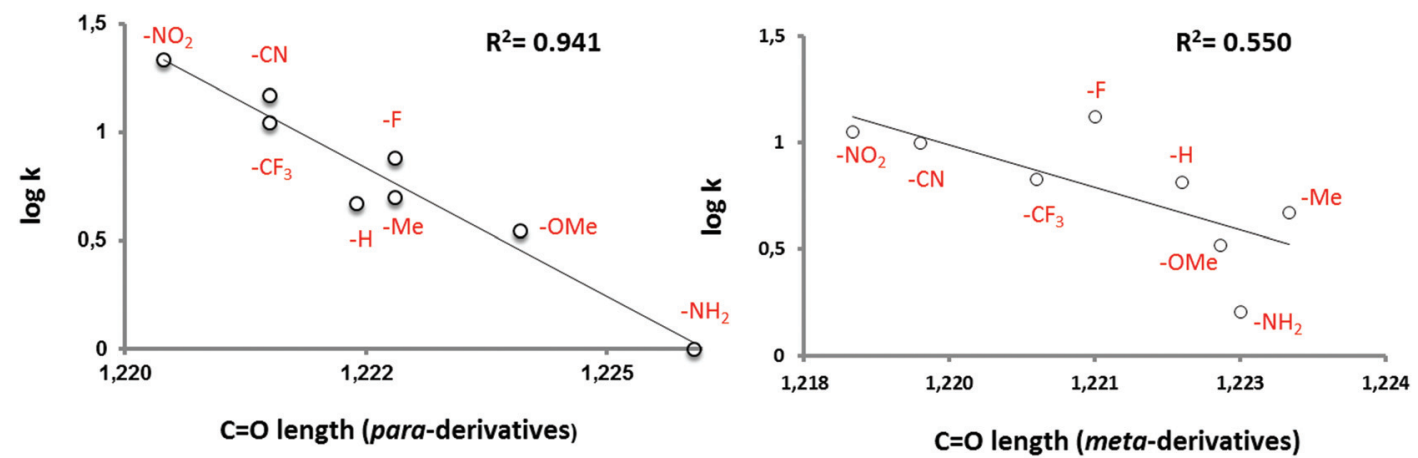

Fig. 3 Correlation between activity and $\mathrm{C}=\mathrm{O}$ length. 
Lactobacillus brevis and Thermoanaerobacter sp., with which the electron withdrawing or donating properties of the substituents did not exert a significant effect. ${ }^{13}$

All these collected data indicate that para-derivatives are reduced with reaction rates apparently not depending on steric interactions of the substrates with the enzyme, whereas steric effects have a (minor) impact only on the reactivity of metaderivatives. The co-occurrence of steric and electronic effects for meta-derivatives was corroborated by the observation that the preferred substrate was 3'-fluoroacetophenone 11 (bearing the less sterically hindered substituent still being an electron withdrawing group).

The occurrence of any substituent in the para- or meta-position favours the delivery of the hydride to the Si face of the ketone, whereas acetophenone 1d predominantly gives $(R)-1$ phenylethanol as a result of an attack on the opposite prostereogenic face. These results are similar to those observed in the reduction of ethyl 2-oxo-2-phenylacetate derivatives with the recombinant alcohol dehydrogenase YMR226c from Saccharomyces cerevisiae: in this case, any type of substituent on the phenyl ring inverted the substrate stereorecognition in the enzyme active site in comparison with the unsubstituted substrate. ${ }^{14}$

Finally, with our tests, we assayed ortho-benzil derivatives (Table 3). ortho-Substituents introduce significant steric effects in the reactivity of acetophenone derivatives, and therefore a simple Hammett's approach to quantify the substituent effects on the reaction center cannot be applied. KRED1-Pglu showed activity only towards 2 '-fluoroacetophenone $1 \mathrm{~s}$ and 2 '-cyanoacetophenone $1 \mathbf{u}$, the substrates bearing electron withdrawing substituents with the lowest steric impact. Low reactivity of sterically demanding ortho-substituted acetophenones is generally observed with a number of recombinant ketoreductases. ${ }^{9,12}$ A notable exception is the Candida tenuis xylose reductase (CtXR), which reduces ortho-substituted acetophenones better than the correspondent para- and meta-derivatives, provided the substituent had both low steric impact and electron withdrawing effect (i.e., $\mathrm{F}, \mathrm{Cl}, \mathrm{Br}) ;{ }^{13} \mathrm{CtXR}$ has a wide binding pocket, able to accommodate a wide variety of substrates, including ortho-substituted acetophenones. ${ }^{25}$

Table 3 Reduction of ortho-substituted acetophenones with KRED1-Pglu

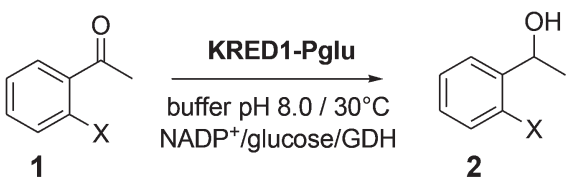

\begin{tabular}{lllccc}
\hline Entry & Substrate & $\mathrm{X}$ & Rel. rate $(\%)^{23}$ & Yield $(\%)^{24}$ & ee (\%) \\
\hline 1 & $\mathbf{1}$ & $\mathrm{NH}_{2}$ & 0 & 0 & - \\
2 & $\mathbf{1 q}$ & $\mathrm{OCH}_{3}$ & 0 & 0 & - \\
3 & $\mathbf{1 r}$ & $\mathrm{CH}_{3}$ & 0 & 0 & - \\
4 & $\mathbf{1 d}$ & $\mathrm{H}$ & 22 & 40 & $95 R$ \\
5 & $\mathbf{1}$ & $\mathrm{F}$ & 76 & 97 & $94 \mathrm{~S}$ \\
6 & $\mathbf{1 t}$ & $\mathrm{CF}_{3}$ & 0 & 0 & - \\
7 & $\mathbf{1 u}$ & $\mathrm{CN}^{2}$ & 82 & 98 & $>98 \mathrm{~S}$ \\
8 & $\mathbf{1 v}$ & $\mathrm{NO}_{2}$ & 0 & 0 & -
\end{tabular}

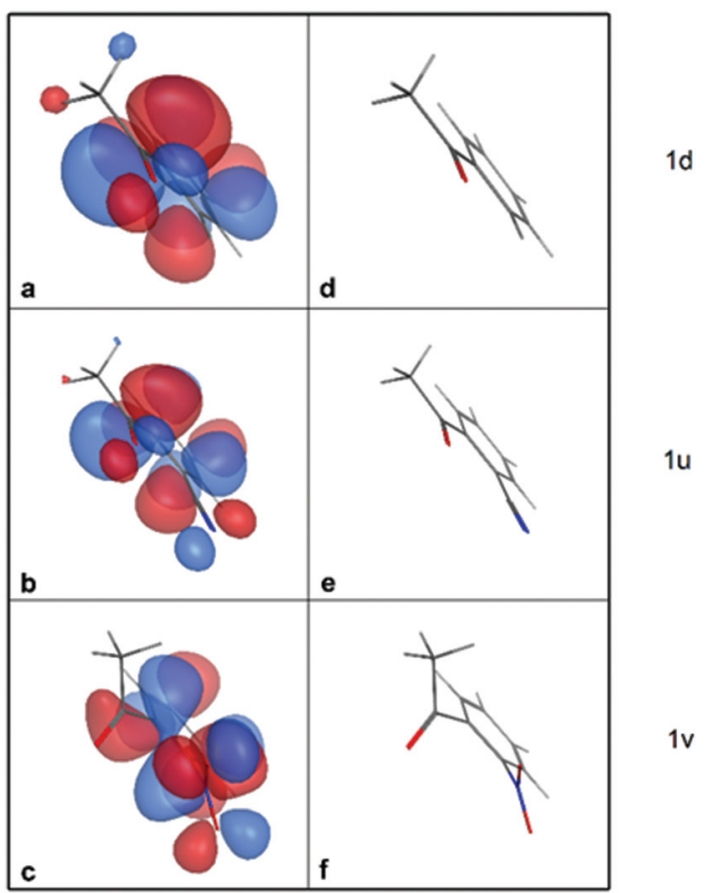

Fig. 4 LUMO density (panels a, b, c) and DFT-based geometry optimization studies (panels $d, e, f$ ) for $1 d, 1 u$ and $1 v$ molecules.

In order to explain the different reactivity for the $\mathrm{C}=\mathrm{O}$ group of 2'-cyanoacetophenone $\mathbf{1} \mathbf{u}$ and 2'-nitroacetophenone $1 \mathbf{v}$, we computed the LUMO frontier molecular orbitals, as shown in Fig. 4, panels b (1u) and c (1v). The LUMO density in 1u (Fig. 4, panel b) is localized on the reactive $\mathrm{C}=\mathrm{O}$, whereas no electronic density correlated with LUMO can be observed, in the same region, for $\mathbf{1} \mathbf{v}$ (Fig. 4, panel c).

In panels e and f, Fig. 4 also shows the results of a DFTbased geometry optimization on 2 '-cyanoacetophenone $1 \mathbf{u}$ and $2^{\prime}$-nitroacetophenone $\mathbf{1 v}$. The almost perfect co-planarity for the $1 \mathbf{u}$ conjugated system (Fig. 4 , panel e), not conserved in $\mathbf{1 v}$ (Fig. 4, panel f) because of the steric hindrance between the $\mathrm{NO}_{2}$ and the $\mathrm{C}=\mathrm{O}$ group, is a further explanation of the different reactivity of these two substrates. Data for acetophenone 1d, are shown in Fig. 4, panels a and d, as a reference. The LUMO frontier molecular orbitals for all the investigated substrates are shown in Fig. S1 $\dagger$. It should be remarked that substrates (such as 1q or 1v), which have an intrinsically low reactivity due to electronic features, were poorly reduced even with the "ortho-specialized" ketoreductase CtXR. ${ }^{13}$

\section{Conclusions}

In conclusion, with this series of comparative tests we showed that a benzil ketoreductase from Pichia glucozyma (KRED1Pglu) is an efficient biocatalyst for the enantioselective reduction of various mono-substituted acetophenones. Reaction rates of meta- and para-derivatives were consistent with the electronic effects described by $\sigma$-Hammett coefficients, 
with the DFT-Mulliken partial charges, and also with the $\mathrm{C}=\mathrm{O}$ length measured on substrates after DFT-geometry optimization. Hence, the electronic properties of the meta- and parasubstituent affected the rate of the reaction, showing that the outcome of the reaction can be predicted and analysed similarly to any other chemical reaction. Reduction of ortho-derivatives occurred only with substrates bearing substituents with low steric impact (i.e., $\mathrm{CN}$ vs. $\mathrm{NO}_{2}$ ). DFT-based geometry optimization and LUMO frontier orbital calculations accounted for the different reactivity of the $\mathrm{C}=\mathrm{O}$ group in the ortho-substituted acetophenone series. (S)-Enantioselectivity with any substrate bearing a substituent on the aromatic ring was observed; notably, only acetophenone gave (R)-1-phenylethanol with high enantioselectivity.

\section{Notes and references}

1 R. Noyori, Angew. Chem., Int. Ed., 2002, 41, 2008.

2 J. C. Moore, D. J. Pollard, B. Kosjek and P. N. Devine, Acc. Chem. Res., 2007, 40, 1412.

3 B. H. Hoff and E. Sundby, Bioorg. Chem., 2013, 51, 31.

4 M. J. Sorgedrager, F. van Rantwijk, G. W. Huisman and R. A. Sheldon, Adv. Synth. Catal., 2008, 350, 2322.

5 E. Garcia-Urdiales, E. Busto, N. Rios-Lombardia, V. GotorFernandez and V. Gotor, ChemCatChem, 2009, 10, 2875.

6 H. Groger, W. Hummel, C. Rollmann, F. Chamouleau, H. Husken, H. Werner, C. Wunderlich, K. Abokitse, K. Drauz and S. Buchholz, Tetrahedron, 2004, 60, 633.

7 I. Lavandera, A. Kern, B. Ferreira-Silva, A. Glieder, S. de Wildeman and W. Kroutil, J. Org. Chem., 2008, 73, 6003.

8 Z. Findrik, D. Vasic-Racki, S. Lutz, T. Daussmann and C. Wandrey, Biotechnol. Lett., 2005, 27, 1087.

9 D. Zhu, B. E. Rios, J. D. Rozzell and L. Hua, Tetrahedron: Asymmetry, 2005, 16, 1541.

10 W. Hummel, Appl. Microbiol. Biotechnol., 1990, 34, 15.
11 P. Hildebrandt, A. Musidlowska, U. T. Bornscheuer and J. Altenbuchner, Appl. Microbiol. Biotechnol., 2002, 59, 483.

12 D. Zhu, Y. Yang and L. Hua, J. Org. Chem., 2006, 26, 4202.

13 M. Vogl, R. Kratzer, B. Nidetzky and L. Breck, Org. Biomol. Chem., 2011, 9, 5863.

14 H. G. Naik, B. Yeniad, C. E. Koning and A. Heise, Org. Biomol. Chem., 2012, 10, 4961.

15 Y. Yang, D. Zhu, T. J. Piegat and L. Hua, Tetrahedron: Asymmetry, 2007, 18, 1799.

16 M. L. Contente, I. Serra, M. Brambilla, I. Eberini, E. Gianazza, V. De Vitis, F. Molinari, P. Zambelli and D. Romano, Appl. Microbiol. Biotechnol., 2016, 100, 193.

17 M. L. Contente, F. Molinari, I. Serra, A. Pinto and D. Romano, Eur. J. Org. Chem., 2016, DOI: 10.1002/ ejoc. 201501557.

18 P. Hoyos, G. Sansottera, M. Ferna, M. F. Molinari, J. V. Sinisterra and A. R. Alcántara, Tetrahedron, 2008, 64, 7929.

19 R. Gandolfi, E. Cesarotti, F. Molinari and D. Romano, Tetrahedron, 2009, 20, 411.

20 M. C. Fragnelli, P. Hoyos, D. Romano, R. Gandolfi, A. R. Alcántara and F. Molinari, Tetrahedron, 2012, 68, 523.

21 C. Forzato, R. Gandolfi, F. Molinari, P. Nitti, G. Pitacco and E. Valentin, Tetrahedron, 2001, 12, 1039.

22 M. L. Contente, F. Molinari, P. Zambelli, V. De Vitis, R. Gandolfi, A. Pinto and D. Romano, Tetrahedron Lett., 2014, 55, 7051.

23 Initial rates were calculated from initial progress curves; $100 \%$ corresponds to the activity of the highest reacting substrate (1h).

24 Analytical yields were determined after $24 \mathrm{~h}$ by GC or HPLC (see the ESI† for details).

25 R. Kratzer, D. K. Wilson and B. Nidetzky, IUBMB Life, 2006, 58, 499. 